\title{
Medial temporal lobe contributions to intra-item associative recognition memory in the aging brain
}

\author{
Marshall Axel Dalton ${ }^{1,2,3}$, Sicong Tu ${ }^{1,2,3}$, Michael Hornberger ${ }^{1,2,3}$, John Russel Hodges ${ }^{1,2,3}$ and \\ Olivier Piguet ${ }^{1,2,3} *$
}

${ }^{1}$ Neuroscience Research Australia, Sydney, NSW, Australia

2 School of Medical Sciences, University of New South Wales, Sydney, NSW, Australia

${ }^{3}$ ARC Centre of Excellence in Cognition and its Disorders, Sydney, NSW, Australia

\section{Edited by:}

Angelica Staniloiu, University of Bielefeld, Germany

Reviewed by:

Aldis Patrick Weible, University of Oregon, USA

Philip Grewe, University Bielefeld, Germany

\section{*Correspondence:}

Olivier Piguet, Neuroscience

Research Australia, Barker Street,

Randwick, NSW 2031, Australia

e-mail: o.piguet@neura.edu.au
Aging is associated with a decline in episodic memory function. This is accompanied by degradation of and functional changes in the medial temporal lobe (MTL) which subserves mnemonic processing. To date no study has investigated age-related functional change in MTL substructures during specific episodic memory processes such as intra-item associative memory. The aim of this study was to characterize age-related change in the neural correlates of intra-item associative memory processing. Sixteen young and 10 older subjects participated in a compound word intra-item associative memory task comprising a measure of associative recognition memory and a measure of recognition memory. There was no difference in performance between groups on the associative memory measure but each group recruited different MTL regions while performing the task. The young group recruited the left anterior hippocampus and posterior parahippocampal gyrus whereas the older participants recruited the hippocampus bilaterally. In contrast, recognition memory was significantly worse in the older subjects. The left anterior hippocampus was recruited in the young group during successful recognition memory whereas the older group recruited a more posterior region of the left hippocampus and showed a more bilateral activation of frontal brain regions than was observed in the young group. Our results suggest a reorganization of the neural correlates of intra-item associative memory in the aging brain.

Keywords: associative memory, medial temporal lobe, hippocampus, perirhinal cortex, aging

\section{INTRODUCTION}

The medial temporal lobes (MTL) contain structures that are crucial for memory processing, such as the hippocampus, perirhinal cortex, and the parahippocampal gyrus. Damage to these structures invariably results in episodic memory impairment (Scoville and Milner, 1957). A growing body of evidence indicates that substructures within the MTL support different elements of episodic memory processing. The hippocampus is implicated in betweendomain associative memory (Mayes et al., 2004; Konkel et al., 2008), that is the high level integration and "binding" of perceptual and conceptual information which are processed and stored in distal brain regions with weak or no direct connectivity with each other. In contrast, extra hippocampal cortical structures such as the perirhinal cortex are implicated in intra-item associative memory (Bussey et al., 2005), that is the unitization of perceptual or conceptual domains represented in closely interacting cortical regions (Davachi, 2006; Mayes et al., 2007). Additional memory functions have also been attributed to these structures. As such, it is argued that the hippocampus is crucial for recollection based recognition memory whereas the perirhinal cortex underlies familiarity based recognition memory (Mayes et al., 2007). In addition, existing evidence shows that memory processing of verbal information or information with a semantic content is lateralized to the anterior regions of the left MTL (Parsons et al., 2006; Ford et al., 2010).
Episodic memory functions decline with age (Christensen, 2001; Nyberg et al., 2003; Ronnlund et al., 2005; Schaie, 2005; Troyer et al., 2011) and young adults consistently outperform older adults on memory tasks which are hypothesized to be hippocampal dependent (Shaw et al., 2006; Head and Isom, 2010; Harris and Wolbers, 2012). MRI and postmortem pathological investigations have shown that among MTL substructures, the hippocampus is particularly sensitive to age-related change (Jack et al., 1998; Raz et al., 2004; Raz and Rodrigue, 2006) with the subiculum and dentate gyrus particularly affected in non-demented older adults (West, 1993; Small et al., 2002). In contrast, the perirhinal cortex appears to undergo little age-related atrophy (Insausti et al., 1998). Functional imaging studies consistently show agerelated reductions in BOLD signal in the MTL during memory task performance (Daselaar et al., 2003; St Jacques et al., 2012).

Investigations of age-related change in the neural correlates of episodic memory retrieval suggest that in parallel with reductions in MTL activation, performance in older adults is associated with the recruitment of additional brain regions, often resulting in a bilateral pattern of activation in the MTL and in frontal cortical regions (Cabeza, 2001; Maguire and Frith, 2003; Giovanello and Schacter, 2012). Whether changes in brain activation as people get older are observed regardless of the type of episodic memory processes involved remains incompletely understood. It is unknown whether age-related changes in activation within and 
beyond the MTL are also found for specific types of associative memory processing such as intra-item associative memory. To address this issue, the present study aimed to: (1) compare intra-item associative recognition memory performance in young and older healthy adults, and (2) establish age-related changes in the neural correlates of intra-item associative recognition memory within the MTL. We used an associative memory task for compound words which included previously studied (targets) and novel items (foils). Some of the novel items were recombined elements of studied components.

We hypothesized that: (1) young adults would outperform older adults on a compound word intra-item associative recognition memory task, (2) successful intra-item associative recognition memory of compound words would recruit greater left $>$ right MTL structures, and (3) the older group would show decreased hippocampal activity compared to the young group.

\section{MATERIALS AND METHODS PARTICIPANTS}

Sixteen, young (12 females) (mean age $=26$ years, range $=21-$ 37 years) and 10 older (5 females) (mean age $=70$ years, range $=62-77$ years) right-handed healthy volunteers were recruited for this study. All were native English speakers with normal or corrected to normal vision and reported no significant neurological or psychiatric disorders on a medical questionnaire. All participants underwent a cognitive screening assessment using the Addenbrooke's Cognitive Examination-Revised (ACE-R). This study was approved by the University of New South Wales Human Ethics Research Committee and all participants provided written informed consent.

\section{STIMULUS MATERIALS}

This study comprised a verbal intra-item associative memory task, adapted from a previous publication (Mayes et al., 2007). Stimuli comprised 100 two- or three-syllable compound words (e.g., gateway, highchair). All stimuli were printed in black on a white background. Three different versions of the task were created and lists were randomly allocated across participants.

\section{PROCEDURES}

The entire experiment was conducted in the scanner and functional magnetic resonance imaging data were acquired during both encoding and test phases. All responses were recorded using an MR compatible button box. At encoding, 60 stimuli were presented on a Phillips LCD monitor one at a time in the center of the screen for $2000 \mathrm{~ms}$, followed by a fixation point for $1000 \mathrm{~ms}$. To ensure optimal attention to the stimuli at encoding, participants were instructed to indicate for each stimulus whether the word was pleasant, unpleasant, or neutral. Before the study phase participants were informed: "You will be shown a number of words. Please tell me if each word evokes a pleasant, unpleasant, or neutral feeling." Encoding was immediately followed by a test phase. At test, 60 stimuli were presented one at time using the same timing procedure. Twenty stimuli were identical to the ones seen at study ("identical"), 20 were novel stimuli not seen at study ("novel"), and 20 stimuli were the combination of two stimuli seen at study ("recombined") (e.g., highchair and gateway at encoding were recombined to become highway at test; Figure 1). Memory for the stimuli seen at study was tested using a yes/no recognition procedure. For each item, participants were instructed to indicate "yes" if they thought the stimulus had been presented at encoding, or "no" if they thought the stimulus had not been seen at encoding. Before the test phase participants were informed: "You will now be shown some more words. For each word please do the following. If you saw the word earlier, press the left button. If you did not see the word earlier, press the right button." Participants were also instructed to respond within the 2000 -ms stimulus presentation window. At test, the order of presentation of identical, recombined, and novel stimuli was pseudo-randomized, in that no items from the same category were seen in succession. In order to become familiarized with the general procedure, participants took part in a practice trial of the encoding and test phases outside the scanner. Participants were not informed about the recombined items at any stage of the experiment.

\section{MR IMAGING PROTOCOL}

MR imaging was acquired on a 3-T Philips Achieva MRI scanner with standard quadrature head coil (16 channels). Functional MR images consisted of the following scanning parameters: 33 slices were collected per image volume covering the whole brain. Scanning parameters for the echo planar imaging (EPI) sequence were as follows: repetition time/echo time (TR/TE) 2000/30 ms; flip angle (FA) $80^{\circ}$; slice thickness $3.5 \mathrm{~mm}$ with a $0-\mathrm{mm}$ interslice gap. For the current task (see below), two encoding runs were collected (75 acquisitions per run). Each encoding run was immediately followed by a test run ( 75 acquisitions per run). In addition, all participants underwent a whole brain T1 coronal orientation, matrix $256 \times 256,180$ slices, $1 \mathrm{~mm}$ isotropic, $\mathrm{TE} / \mathrm{TR}=2.5 / 5.4 \mathrm{~ms}$, FA $\alpha=8^{\circ}$.

\section{fMRI DATA ANALYSIS}

Images were analyzed using fMRI Expert Analysis Tool (FEAT) version 5.98, a part of FSL (FMRIB's Software Library, www.fmrib. ox.ac.uk/fsl). Pre-processing of each individual's fMRI dataset included: removal of non-brain structures from the T1 structural scans using Brain Extraction Tool (BET), motion correction using MCFLIRT, non-brain structures were removed from the echoplanar imaging volumes using BET, spatial smoothing using a Gaussian Kernel of FWHM $5 \mathrm{~mm}$; mean based intensity normalization of the entire $4 \mathrm{D}$ dataset by the same multiplicative factor; high pass temporal filtering (Gaussian weighted leastsquares straight line fitting, with $\sigma=100 \mathrm{~s}$ ). Time series statistical analysis was performed using FILM with local autocorrelation correction. Functional scans were registered to the high resolution T1 structural scan per participant and to the standard Montreal Neurological Institute (MNI 152) standard space template image using affine registration with FLIRT. Coordinates $(x, y, z)$ of activation are reported in MNI space.

For each subject a fixed effects model was used to estimate effects for each stimulus type. The following contrasts were modeled: identical item hits vs. correct novel item rejections and identical item hits vs. correct recombined item rejections. The resulting data were then entered into a mixed effects higher level analysis to investigate activity across participants for each comparison. 


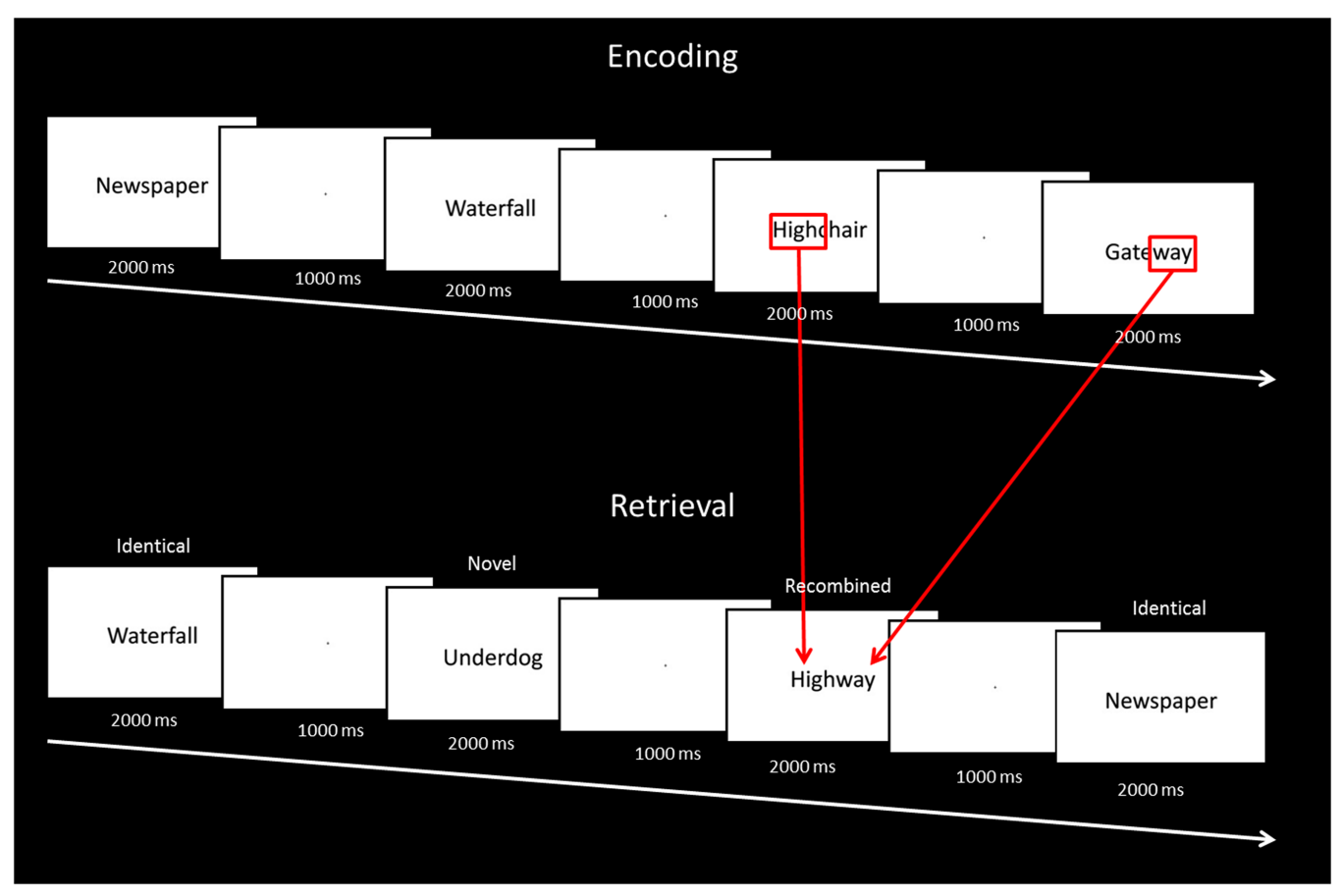

FIGURE 1 | Description of the study and test phases of the intra-item associative memory task.

$Z$ statistic images were thresholded using clusters determined by $Z>2.3$ and an uncorrected cluster significance threshold of $p<0.01$. In addition, the $\%$ signal change within each cluster was extracted for each group.

\section{RESULTS}

\section{BEHAVIORAL}

Performance on the general cognitive measure ACE-R was not significantly different between the young and older groups. Corrected recognition memory for identical compound words (i.e., hits-false alarms) differed significantly between groups, with the young participants outperforming their older counterparts [85 and $58 \%$ respectively; $t(24)=4.568, p=<0.001$ ]. In contrast, no significant group differences were found in identifying either recombined [ 57 and $42 \%$ respectively; $t(24)=1.630, p=0.116$ ] or novel compound words [95 and $91 \%$ respectively; $t(24)=1.182$, $p=0.249$ ] (Figure 2). Investigating within group performance, we found significant differences in accuracy between identical and recombined items [ 85 vs. $57 \%, t(30)=4.951, p=<0.001$ ], between recombined and novel items [ 57 vs. 95\%, $t(30)=-7.535$, $p=<0.001]$, and between identical and novel items [85 and $95 \%, t(30)=-3.162, p=0.004]$ in the young group. In contrast, in the older group, there was a significant difference in accuracy between recombined and novel items [42 and 91\%, $t(18)=-5.247, p=<0.001]$ and identical and novel items [58 and $91 \%, t(18)=-4.693, p=<0.001]$ but no significant difference between identical and recombined items [58 and 48\%, $t(18)=1.540, p=0.141]$.

Analyses on response latency revealed that in the young group, correct responses to recombined items $(1160 \pm 271 \mathrm{~ms})$ were

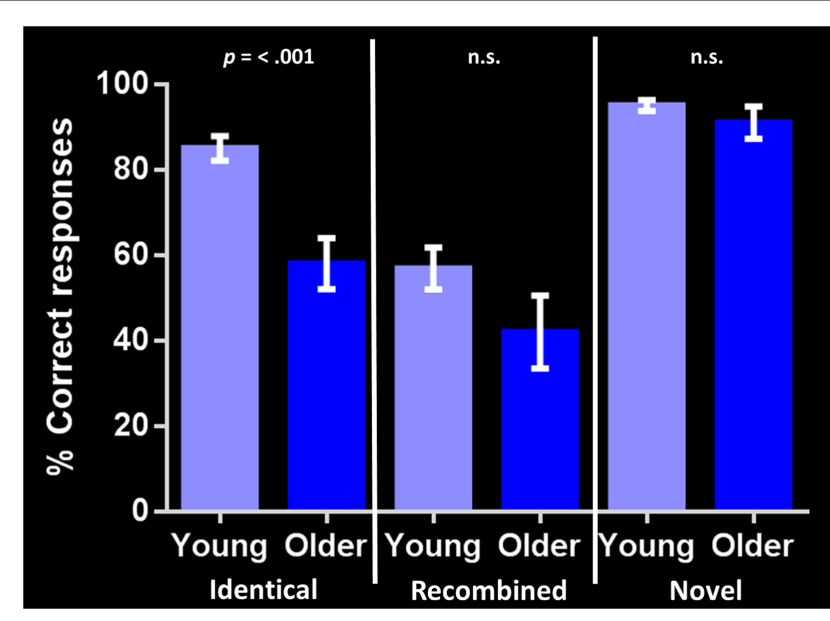

FIGURE 2 | Corrected recognition memory performance for each stimulus condition in young and older adults (i.e., hits-false alarms). The bar chart shows the percent correct response for each stimulus type (identical, recombined, novel). Error bars represent the standard error of the mean. $p=<0.001$ indicates significant difference between young and older adult groups. n.s., indicates non-significant difference.

significantly slower than responses to identical $[1003 \pm 243 \mathrm{~ms}$, $t(681)=-7.96, p=<0.001]$ and novel $[1008 \pm 262 \mathrm{~ms}, t(683)=$ 7.43, $p<0.001]$ items. No significant difference was found in response latency between identical and novel items. In the older group, correct responses to recombined items $(1246 \pm 285 \mathrm{~ms})$ were significantly slower than responses to 
identical [1112 $\pm 280 \mathrm{~ms}, t(332)=-4.327, p=<0.001]$ and novel $[1084 \pm 280 \mathrm{~ms}, t(334)=5.233, p=<0.001]$ items. No significant difference was found in response latency between identical and novel items.

The young group responded significantly faster than the older group for all item types: identical [young: $1003 \pm 243 \mathrm{~ms}$; older: $1112 \pm 278 \mathrm{~ms} ; t(521)=-4.6, p=<0.001]$, recombined [young: $1159 \pm 271 \mathrm{~ms}$; older: $1246 \pm 285 \mathrm{~ms} ; t(494)=-3.3, p=$ $<0.001$ ] , and novel [young: $1008 \pm 262 \mathrm{~ms}$; older: $1084 \pm 280 \mathrm{~ms}$; $t(523)=-3.08, p=0.002]$. The number of late responses (i.e., $>2000 \mathrm{~ms}$ ) did not differ between the young and older groups.

\section{IMAGING}

Associative recognition memory (identical vs. recombined contrast) In the young group, the identical vs. recombined rejection contrast revealed significant activation within the MTL in two regions of the left hippocampus $(x=-36, y=-24, z=-14$ and $x=-26$, $y=-14, z=-22)$ and in the left posterior parahippocampal gyrus $(x=-26, y=-38, z=-10)$ (Figure 3A; Table 1). In addition, significant clusters of activation were also observed in the middle frontal gyrus, putamen, lateral occipital cortex, precentral gyrus, posterior cingulate gyrus, supramarginal gyrus, and frontal pole bilaterally (Table $\mathbf{1}$ ).

The same contrast in the older group revealed significant activation within the MTL in the hippocampus bilaterally (right: $x=36$, $y=-18, z=-16$ and left: $x=-30, y=-20, z=-14)$ and the left perirhinal cortex $(x=-32, y=-14, z=-32)$ (Figure 3B; Table 1). Additional significant activation was observed in the insular cortex, posterior cingulate cortex, occipital fusiform gyrus, and the middle frontal gyrus (Table $\mathbf{1}$ ).

\section{Recognition memory (identical vs. novel contrast)}

In the young adult group, the identical vs. novel item contrast revealed a significant cluster of activation in the left anterior hippocampus ( $x=-28, y=-14, z=-24$; Figure 4A; Table 2). In addition, significant activation in were found in the lateral occipital cortex, middle frontal gyrus, frontal pole, thalamus, superior frontal gyrus, and precuneus (Table 2).

In the older group, the same contrast revealed a small cluster of activation within the left hippocampus $(x=-14, y=-16$, $z=-22$; Figure 4B; Table 2). Broad bilateral activation was also present in the middle frontal gyrus and the frontal pole, as well as in the angular gyrus, superior frontal gyrus, lateral occipital cortex, precentral gyrus, paracingulate gyrus, and the supramarginal gyrus (Table 2).

\section{DISCUSSION}

This study identified the neural correlates of associative recognition memory for compound words in young and older healthy adults. Although performance in associative recognition memory was matched between groups, young and older adults differed in the location and extent of MTL involvement supporting task

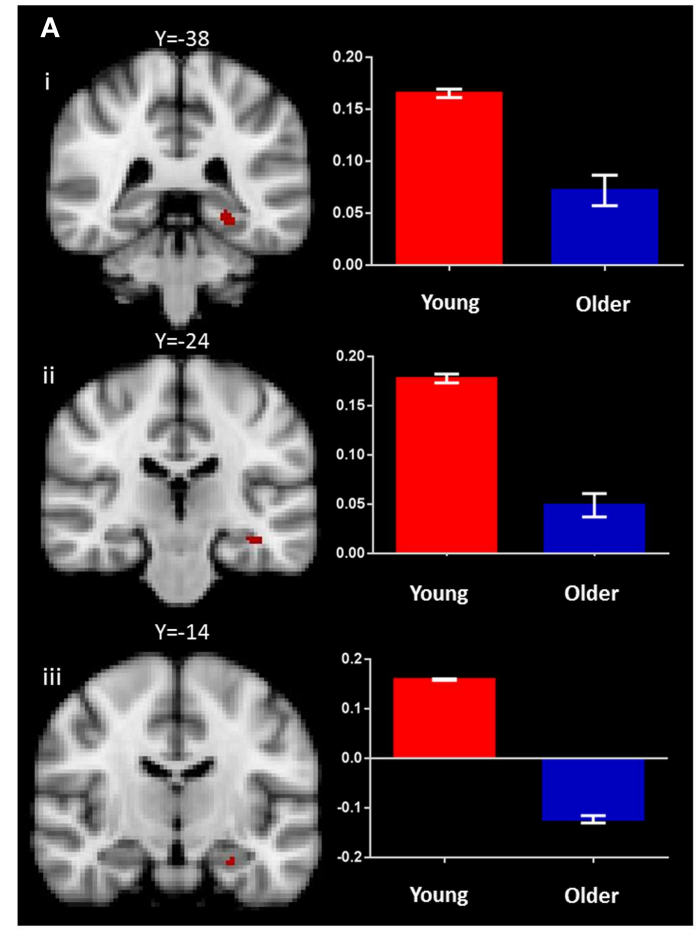

FIGURE 3 | Regions of increased BOLD signal associated with associative recognition memory (identical > recombined rejection) for: (A) young: (i) left posterior parahippocampal gyrus $(x=-26$, $y=-38, z=-10)$; (ii,iii) left anterior hippocampus $(x=-36$, $y=-24, z=-14)(x=-26, y=-14, z=-22)$ and (B) old: (i) right

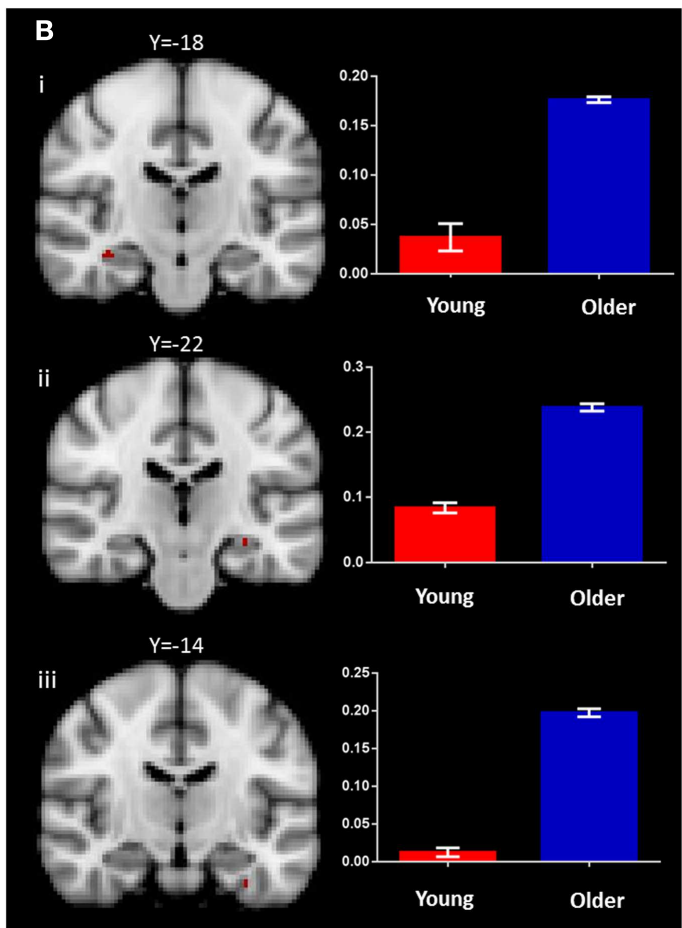

anterior hippocampus ( $x=36, y=-18, z=-16)$; (ii) left anterior hippocampus $(x=-30, y=-22, z=-14)$; (iii) left anterior parahippocampal gyrus $(x=-32, y=-14, z=-32)$ subjects. Graph depicts mean percent signal change associated with each group within the region of interest. 
Table 1 | BOLD signal increase for the contrast of identical hits > correct recombined rejection in young and older participants.

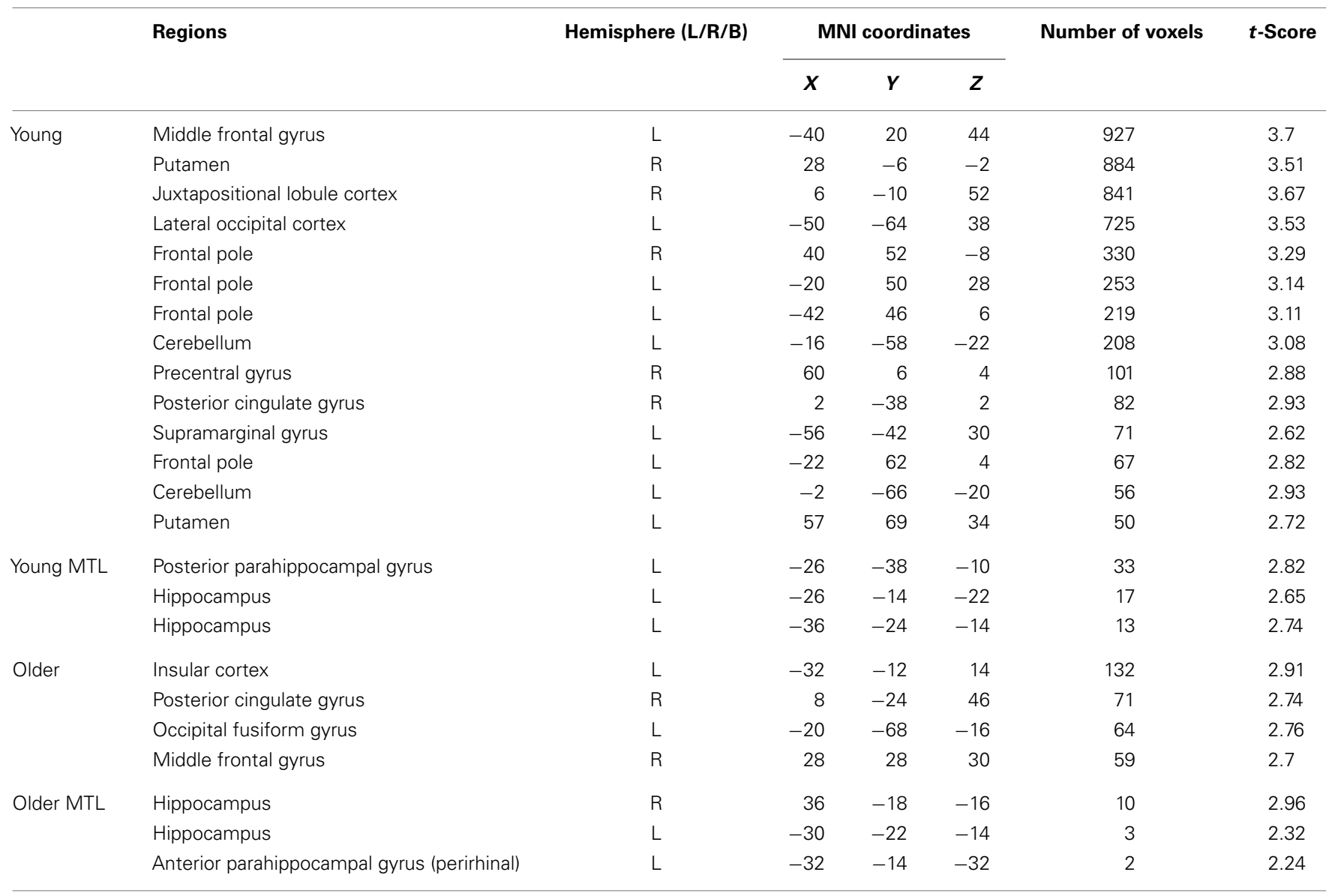

Results are reported at $p<0.001$ uncorrected with at least 50 contiguous voxels.

performance. Young adults showed a left lateralized activation involving the anterior hippocampus and posterior parahippocampal gyrus. In contrast, older participants revealed hippocampal involvement bilaterally. In addition to these MTL regions, associative memory performance was also associated with increased activity in a number of cortical and subcortical regions including the middle frontal gyrus, putamen, lateral occipital cortex, precentral gyrus, posterior cingulate gyrus, supramarginal gyrus, and frontal pole in the young adults, as well as the insular cortex, posterior cingulate, occipital fusiform, and middle frontal gyrus in the older adults.

These results suggest a reorganization in the neural correlates of associative recognition memory for compound words with age. Imaging findings from the young group align well with the view that verbal memory processing is supported by a left lateralized distributed network involving anterior regions of the MTL (Binder et al., 2003; Daselaar et al., 2003; Parsons et al., 2006; Ford et al., 2010). More specifically, we found left anterior hippocampal and posterior parahippocampal gyrus activation in this group. A previous study utilized a similar task to the one used in the present study to investigate associative memory processing in young adults and reported recruitment of the left perirhinal cortex (Ford et al., 2010). We found no evidence of left perirhinal cortex recruitment in the young group but did observe a small left perirhinal cortex cluster in the older group. Importantly, bilateral hippocampus recruitment was also present in the older group. To our knowledge, this is the first observation of age-related functional change in the neural correlates of associative recognition memory for compound words. Age-related changes affecting the laterality of MTL activation have previously been observed in functional imaging studies of autobiographical memory retrieval, with predominant left hippocampus recruitment found in young adults compared to bilateral activation in older adults (Maguire and Frith, 2003). We observed a similar age-related left-right shift in MTL activation during successful associative recognition memory for compound words. Involvement of the left anterior hippocampus was observed only in the young group. In the older group, activation of more posterior regions of the hippocampus bilaterally supporting memory performance was found instead.

In addition to changes in MTL activity, we also observed changes in a number of cortical and subcortical brain regions, which have been previously implicated in verbal memory processing, verbal fluency, and naming of objects (Valenstein et al., 1987; Petrides et al., 1993; Salmon et al., 1996; Rosen et al., 2000; Chouinard et al., 2009; Bokde et al., 2010; Lim et al., 2012; Thames et al., 2012; Costa et al., 2013). Recruitment of some of these 

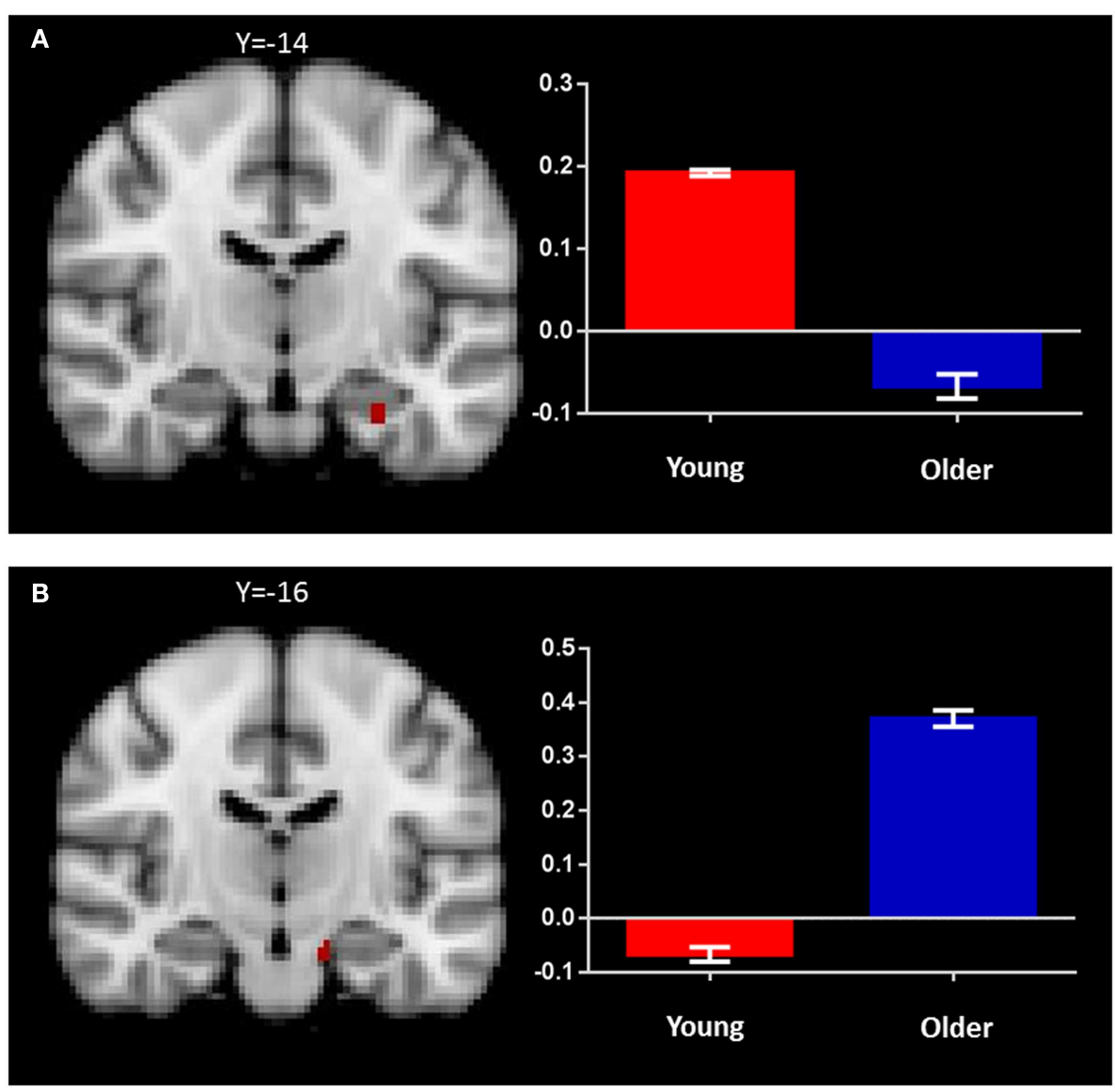

FIGURE 4 | Regions of increased BOLD signal associated with recognition memory (identical > novel) for: (A) young: left anterior hippocampus $(x=-\mathbf{2 8}, y=-\mathbf{1 4}, z=-\mathbf{2 4})$ and $(B)$ old $(x=-14, y=-16, z=-22)$ subjects. Graph depicts mean percent signal change associated with each group within the region of interest.

regions was age specific. Activation in the putamen, frontal pole, supramarginal gyrus, and lateral occipital cortex was found in the young group only. Regions of activation observed only in the older group included the insular cortex and fusiform gyrus, again regions that have been previously implicated in verbal memory (Paulesu et al., 1993; Grasby et al., 1994; Manes et al., 1999) although the fusiform cortex is generally considered to be involved in memory processing of non-verbal pictorial rather than verbal stimuli (Kim, 2011).

In contrast to associative recognition memory, recognition memory (i.e., correct identification of identical stimuli) differed between groups, with older adults experiencing greater difficulty than young participants on this component of the task. As anticipated, the neural correlates of recognition memory also differed between groups. During this task, young adults recruited the left anterior hippocampus whereas older adults showed involvement of the left hippocampus more posteriorly with additional recruitment of frontal cortical regions bilaterally. In other words, older adults were unable to maintain a level of performance similar to that of young adults despite the recruitment of additional brain regions. The increase in bilateral brain activation with age during memory retrieval has been reported previously and appears to reflect a compensatory process (Reuter-Lorenz and Cappell, 2008; Cappell et al., 2010). Although recognition of recombined stimuli is inherently more difficult than that of identical stimuli, we found an age difference on the recognition performance for the identical but not the recombined component of the task. Whilst we observed a drop in response accuracy between identical and recombined stimuli for the young group, performance in the older group between conditions remained relatively unchanged. Two explanations may underlie this unanticipated result. First, evidence indicates that older adults tend to show a more liberal response pattern in recognition memory tasks compared to young adults (Huh et al., 2006). Frequency of false alarms during recognition memory tasks also tends to rise with task difficulty, regardless of age. Indeed, in this study, the young group showed higher false alarms in the recombined than the identical conditions. The frequency of false alarms, however, remained stable in the older group. This may have been due to the task instructions. Here, correct responses to the instructions ("Have you seen this word before?") necessitated opposing behaviors depending on the stimulus types: "yes" responses to identical stimuli, but 
Table 2 | BOLD signal increase for the contrast of identical hits $>$ novel hits in young and older participants.

\begin{tabular}{|c|c|c|c|c|c|c|c|}
\hline & Regions & Hemisphere (L/R/B) & \multicolumn{3}{|c|}{ MNI coordinates } & Number of voxels & $t$-Score \\
\hline & Middle frontal gyrus & $\mathrm{L}$ & -46 & 18 & 34 & 274 & 3.16 \\
\hline & Frontal pole & $\mathrm{R}$ & 44 & 36 & 20 & 138 & 3.03 \\
\hline & Lateral occipital cortex & $\mathrm{R}$ & 36 & -72 & 46 & 126 & 2.74 \\
\hline & Superior frontal gyrus & $\mathrm{R}$ & 26 & 30 & 54 & 71 & 2.95 \\
\hline & Juxtapositional lobule cortex & $\mathrm{R}$ & 6 & -8 & 48 & 70 & 3.19 \\
\hline & Frontal pole & $\mathrm{R}$ & 10 & 56 & 18 & 68 & 2.72 \\
\hline & Precuneus cortex & $\mathrm{R}$ & 4 & -56 & 34 & 61 & 2.64 \\
\hline Young MTL & Hippocampus & $\mathrm{L}$ & -28 & -14 & -24 & 14 & 2.67 \\
\hline \multirow{10}{*}{ Older } & Middle frontal gyrus & $\mathrm{R}$ & 36 & 10 & 48 & 245 & 2.9 \\
\hline & Frontal pole & $\mathrm{L}$ & -38 & 40 & 6 & 207 & 2.89 \\
\hline & Middle frontal gyrus & $L$ & -52 & 30 & 24 & 201 & 2.86 \\
\hline & Frontal pole & $\mathrm{L}$ & -10 & 64 & 20 & 148 & 2.93 \\
\hline & Middle frontal gyrus & $\mathrm{R}$ & 44 & 26 & 24 & 83 & 2.81 \\
\hline & Frontal pole & $\mathrm{R}$ & 20 & 52 & 12 & 82 & 2.72 \\
\hline & Precentral gyrus & $\mathrm{R}$ & 46 & 4 & 26 & 79 & 2.82 \\
\hline & Frontal pole & $L$ & -18 & 66 & 0 & 76 & 2.89 \\
\hline & Paracingulate gyrus & L & -12 & 44 & 14 & 64 & 2.63 \\
\hline & Supramarginal gyrus & $L$ & -60 & -46 & 40 & 50 & 2.7 \\
\hline
\end{tabular}

Results are reported at $p<0.001$ uncorrected with at least 50 contiguous voxels.

"no" responses to recombined stimuli. It is therefore plausible that the response type required in this condition (i.e., having to reject correctly identified recombined stimuli) may have counterbalanced the liberal bias generally observed in the older group and reduced the false alarm responses, thus reducing the betweengroup difference. Second, close inspection of individual response profiles revealed that three young participants scored at least 2 SDs below the group mean for the recombined condition, contributing to the lack of group difference ${ }^{1}$.

The group difference in the patterns of brain activation may represent a functional reorganization and compensation for the decreased efficiency in hippocampal recruitment found as individuals get older. Hippocampal volumes decrease with healthy aging (Raz and Rodrigue, 2006) and are predictive of explicit memory performance in subjects over the age of 60 (Raz et al., 1998; Lye et al., 2004). Loss of synaptic density, rather than neuronal loss, is the main contributor to the volume reduction (Rosenzweig and Barnes, 2003; Burke and Barnes, 2006). Not all hippocampal regions undergo the same changes, however, with anterior regions

${ }^{1}$ Indeed, re-analysis of the behavioral data when these three outliers from the young group are excluded result in a significant between-group difference for the recombined condition. appearing to be more resilient to age-related degradation than posterior regions (Driscoll et al., 2003). Within this framework, the recruitment of posterior regions of the hippocampus in the older group remains to be investigated further. Age-related reductions in hippocampal activation have been reported during a number of memory tasks using differing methodologies such as encoding of nouns (Daselaar et al., 2003), autobiographical memory retrieval (St Jacques et al., 2012), working memory for complex scenes (Park et al., 2003) and relational encoding in working memory (Mitchell et al., 2000). In addition, memory performance is also affected by the integrity of the connections between MTL and surrounding structures (Hornberger et al., 2012). The reduced activation in the left anterior hippocampus found in the older group accords well with these findings.

The perirhinal cortex and parahippocampal gyrus are components of two dissociable cortical networks with the perirhinal cortex contributing to memory for item information and the parahippocampal gyrus contributing to memory for context (Ranganath and Ritchey, 2012). The parahippocampal gyrus has also been implicated in episodic simulation (Addis et al., 2009). As such, the difference in brain activation may also indicate the use of different mnemonic strategies in the young and older groups. The increased posterior parahippocampal gyrus activation and lack of 
perirhinal cortex activation in the young group possibly reflects the reliance on mnemonic techniques such as visualization or mental elaboration in this group (e.g., Kondo et al., 2005). In post task debriefings, participants commonly mentioned the use of visualization and association as mnemonic tools to help remember each item. Whether the use of such strategies differed between the two groups was not formally investigated.

Arguably, our findings need to be taken with caution in the view of the uncorrected results reported here. Nevertheless, while the activation clusters in the MTL may appear small, they are in line with those found previously (Staresina and Davachi, 2006). These potential limitations notwithstanding, we have shown that associative recognition memory for compound words is associated with left lateralized MTL structures in young individuals and bilateral MTL structures in their older counterparts. We provide evidence for a functional reorganization of the neural correlates of associative memory processing in the aging brain. These findings have important implications for theoretical models of associative memory processing, in that they support the view that different regions of the MTL are capable of supporting associative recognition memory for verbal stimuli in different stages of life.

\section{ACKNOWLEDGMENTS}

This work was supported in part by an Australian Research Council (ARC) Discovery Project (DP1093279); the ARC Centre of Excellence in Cognition and its Disorders (CE110001021); Marshall Axel Dalton is supported by an Australian Rotary Health award. Sicong Tu is supported by Alzheimer's Australia Dementia Research Foundation and NHMRC awards. Michael Hornberger is supported by an ARC Research Fellowship (DP110104202); Olivier Piguet is supported by a National Health and Medical Research Council of Australia Career Development Fellowship (NHMRC, APP 1022684); these sources had no role in the study design, collection, analyses, and interpretation of data, writing of the manuscript, or in the decision to submit the paper for publication.

\section{REFERENCES}

Addis, D. R., Pan, L., Vu, M.-A., Laiser, N., and Schacter, D. L. (2009). Constructive episodic simulation of the future and the past: distinct subsystems of a core brain network mediate imagining and remembering. Neuropsychologia 47, 2222-2238. doi:10.1016/j.neuropsychologia.2008.10.026

Binder, J. R., McKiernan, K. A., Parsons, M. E., Westbury, C. F., Possing, E. T., Kaufman, J. N., et al. (2003). Neural correlates of lexical access during visual word recognition. J. Cogn. Neurosci. 15, 372-393. doi:10.1162/089892903321593108

Bokde, A. L., Karmann, M., Born, C., Teipel, S. J., Omerovic, M., Ewers, M., et al. (2010). Altered brain activation during a verbal working memory task in subjects with amnestic mild cognitive impairment. J. Alzheimers Dis. 21, 103-118. doi:10.3233/JAD-2010-091054

Burke, S. N., and Barnes, C. A. (2006). Neural plasticity in the ageing brain. Nat. Rev. Neurosci. 7, 30-40. doi:10.1038/nrn1809

Bussey, T. J., Saksida, L. M., and Murray, E. A. (2005). The perceptualmnemonic/feature conjunction model of perirhinal cortex function. Q. J. Exp. Psychol. B 58, 269-282. doi:10.1080/02724990544000004

Cabeza, R. (2001). Cognitive neuroscience of aging: contributions of functional neuroimaging. Scand. J. Psychol. 42, 277-286. doi:10.1111/1467-9450.00237

Cappell, K. A., Gmeindl, L., and Reuter-Lorenz, P. A. (2010). Age differences in prefontal recruitment during verbal working memory maintenance depend on memory load. Cortex 46, 462-473. doi:10.1016/j.cortex.2009.11.009
Chouinard, P. A., Whitwell, R. L., and Goodale, M. A. (2009). The lateral-occipital and the inferior-frontal cortex play different roles during the naming of visually presented objects. Hum. Brain Mapp. 30, 3851-3864. doi:10.1002/hbm.20812

Christensen, H. (2001). What cognitive changes can be expected with normal ageing? Aust. N. Z. J. Psychiatry 35, 768-775. doi:10.1046/j.1440-1614.2001.00966.x

Costa, A., Oliveri, M., Barban, F., Bonni, S., Koch, G., Caltagirone, C., et al. (2013). The right frontopolar cortex is involved in visual-spatial prospective memory. PLoS ONE 8:e56039. doi:10.1371/journal.pone.0056039

Daselaar, S. M., Veltman, D. J., Rombouts, S. A., Raaijmakers, J. G., and Jonker, C. (2003). Neuroanatomical correlates of episodic encoding and retrieval in young and elderly subjects. Brain 126, 43-56. doi:10.1093/brain/awg005

Davachi, L. (2006). Item, context and relational episodic encoding in humans. Curr. Opin. Neurobiol. 16, 693-700. doi:10.1016/j.conb.2006.10.012

Driscoll, I., Hamilton, D. A., Petropoulos, H., Yeo, R. A., Brooks, W. M., Baumgartner, R. N., et al. (2003). The aging hippocampus: cognitive, biochemical and structural findings. Cereb. Cortex 13, 1344-1351. doi:10.1093/cercor/bhg081

Ford, J. H., Verfaellie, M., and Giovanello, K. S. (2010). Neural correlates of familiarity-based associative retrieval. Neuropsychologia 48, 3019-3025. doi:10. 1016/j.neuropsychologia.2010.06.010

Giovanello, K. S., and Schacter, D. L. (2012). Reduced specificity of hippocampal and posterior ventrolateral prefrontal activity during relational retrieval in normal aging. J. Cogn. Neurosci. 24, 159-170. doi:10.1162/jocn_a_00113

Grasby, P. M., Frith, C. D., Friston, K. J., Simpson, J., Fletcher, P. C., Frackowiak, R. S., et al. (1994). A graded task approach to the functional mapping of brain areas implicated in auditory-verbal memory. Brain 117(Pt 6), 1271-1282. doi:10.1093/brain/117.6.1271

Harris, M. A., and Wolbers, T. (2012). Ageing effects on path integration and landmark navigation. Hippocampus 22, 1770-1780. doi:10.1002/hipo.22011

Head, D., and Isom, M. (2010). Age effects on wayfinding and route learning skills. Behav. Brain Res. 209, 49-58. doi:10.1016/j.bbr.2010.01.012

Hornberger, M., Wong, S., Tan, R., Irish, M., Piguet, O., Kril, J., et al. (2012). In vivo and post-mortem memory circuit integrity in frontotemporal dementia and Alzheimer's disease. Brain 135, 3015-3025. doi:10.1093/brain/aws239

Huh, T. J., Kramer, J. H., Gazzaley, A., and Delis, D. C. (2006). Response bias and aging on a recognition memory task. J. Int. Neuropsychol. Soc. 12, 1-7. doi:10.1017/S1355617706060024

Insausti, R., Juottonen, K., Soininen, H., Insausti, A. M., Partanen, K., Vainio, P., et al. (1998). MR volumetric analysis of the human entorhinal, perirhinal, and temporopolar cortices. AJNR Am. J. Neuroradiol. 19, 659-671.

Jack, C. R. Jr., Petersen, R. C., Xu, Y., O’Brien, P. C., Smith, G. E., Ivnik, R. J., et al. (1998). Rate of medial temporal lobe atrophy in typical aging and Alzheimer's disease. Neurology 51, 993-999. doi:10.1212/WNL.51.4.993

Kim, H. (2011). Neural activity that predicts subsequent memory and forgetting: a meta-analysis of $74 \mathrm{fMRI}$ studies. Neuroimage 54, 2446-2461. doi:10.1016/j. neuroimage.2010.09.045

Kondo, Y., Suzuki, M., Mugikura, S., Abe, N., Takahashi, S., Iijima, T., et al. (2005). Changes in brain activation associated with use of a memory strategy: a functional MRI study. Neuroimage 24, 1154-1163. doi:10.1016/j.neuroimage.2004. 10.033

Konkel, A., Warren, D. E., Duff, M. C., Tranel, D. N., and Cohen, N. J. (2008). Hippocampal amnesia impairs all manner of relational memory. Front. Hum. Neurosci. 2:15. doi:10.3389/neuro.09.015.2008

Lim, T. S., Hong, Y. H., Choi, J. Y., Kim, H. S., and Moon, S. Y. (2012). Functional investigation of bilateral posterior cingulate gyri using multivoxel MR spectroscopy. Eur. Neurol. 67, 279-286. doi:10.1159/000336834

Lye, T. C., Piguet, O., Grayson, D. A., Creasey, H., Ridley, L. J., Bennett, H. P., et al. (2004). Hippocampal size and memory function in the ninth and tenth decades of life: the Sydney Older Persons Study. J. Neurol. Neurosurg. Psychiatry 75, 548-554. doi:10.1136/jnnp.2003.010223

Maguire, E. A., and Frith, C. D. (2003). Aging affects the engagement of the hippocampus during autobiographical memory retrieval. Brain 126, 1511-1523. doi:10.1093/brain/awg157

Manes, F., Springer, J., Jorge, R., and Robinson, R. G. (1999). Verbal memory impairment after left insular cortex infarction. J. Neurol. Neurosurg. Psychiatry 67, 532-534. doi:10.1136/jnnp.67.4.532

Mayes, A., Montaldi, D., and Migo, E. (2007). Associative memory and the medial temporal lobes. Trends Cogn. Sci. 11, 126-135. doi:10.1016/j.tics.2006.12.003 
Mayes, A. R., Holdstock, J. S., Isaac, C. L., Montaldi, D., Grigor, J., Gummer, A., et al. (2004). Associative recognition in a patient with selective hippocampal lesions and relatively normal item recognition. Hippocampus 14, 763-784. doi:10.1002/hipo.10211

Mitchell, K. J., Johnson, M. K., Raye, C. L., and D’Esposito, M. (2000). fMRI evidence of age-related hippocampal dysfunction in feature binding in working memory. Brain Res. Cogn. Brain Res. 10, 197-206. doi:10.1016/S0926-6410(00)00029-X

Nyberg, L., Maitland, S. B., Ronnlund, M., Backman, L., Dixon, R. A., Wahlin, A., et al. (2003). Selective adult age differences in an age-invariant multifactor model of declarative memory. Psychol. Aging 18, 149-160. doi:10.1037/0882-7974.18. 1.149

Park, D. C., Welsh, R. C., Marshuetz, C., Gutchess, A. H., Mikels, J., Polk, T. A., et al. (2003). Working memory for complex scenes: age differences in frontal and hippocampal activations. J. Cogn. Neurosci. 15, 1122-1134. doi:10.1162/ 089892903322598094

Parsons, M. W., Haut, M. W., Lemieux, S. K., Moran, M. T., and Leach, S. G. (2006). Anterior medial temporal lobe activation during encoding of words: FMRI methods to optimize sensitivity. Brain Cogn. 60, 253-261. doi:10.1016/j.bandc.2005. 07.010

Paulesu, E., Frith, C. D., and Frackowiak, R. S. (1993). The neural correlates of the verbal component of working memory. Nature 362, 342-345. doi:10.1038/ 362342a0

Petrides, M., Alivisatos, B., Meyer, E., and Evans, A. C. (1993). Functional activation of the human frontal cortex during the performance of verbal working memory tasks. Proc. Natl. Acad. Sci. U.S.A. 90, 878-882. doi:10.1073/pnas.90.3.878

Ranganath, C., and Ritchey, M. (2012). Two cortical systems for memory-guided behaviour. Nat. Rev. Neurosci. 13, 713-726. doi:10.1038/nrn3338

Raz, N., Gunning-Dixon, F. M., Head, D., Dupuis, J. H., and Acker, J. D. (1998). Neuroanatomical correlates of cognitive aging: evidence from structural magnetic resonance imaging. Neuropsychology 12, 95-114. doi:10.1037/08944105.12.1.95

Raz, N., and Rodrigue, K. M. (2006). Differential aging of the brain: patterns, cognitive correlates and modifiers. Neurosci. Biobehav. Rev. 30, 730-748. doi:10.1016/ j.neubiorev.2006.07.001

Raz, N., Rodrigue, K. M., Head, D., Kennedy, K. M., and Acker, J. D. (2004). Differential aging of the medial temporal lobe: a study of a five-year change. Neurology 62, 433-438. doi:10.1212/01.WNL.0000106466.09835.46

Reuter-Lorenz, P. A., and Cappell, K. A. (2008). Neurocognitive aging and the compensation hypothesis. Curr. Dir. Psychol. Sci. 17, 177-182. doi:10.1111/j.14678721.2008.00570.x

Ronnlund, M., Nyberg, L., Backman, L., and Nilsson, L. G. (2005). Stability, growth, and decline in adult life span development of declarative memory: cross-sectional and longitudinal data from a population-based study. Psychol. Aging 20, 3-18. doi:10.1037/0882-7974.20.1.3

Rosen, H. J., Ojemann, J. G., Ollinger, J. M., and Petersen, S. E. (2000). Comparison of brain activation during word retrieval done silently and aloud using fMRI. Brain Cogn. 42, 201-217. doi:10.1006/brcg.1999.1100

Rosenzweig, E. S., and Barnes, C. A. (2003). Impact of aging on hippocampal function: plasticity, network dynamics, and cognition. Prog. Neurobiol. 69, 143-179. doi:10.1016/S0301-0082(02)00126-0
Salmon, E., Van der Linden, M., Collette, F., Delfiore, G., Maquet, P., Degueldre, C., et al. (1996). Regional brain activity during working memory tasks. Brain $119(\mathrm{Pt}$ 5), 1617-1625. doi:10.1093/brain/119.5.1617

Schaie, K. W. (2005). Developmental Influences on Adult Intelligence: The Seattle Longitudinal Study. New York: Oxford University Press.

Scoville, W. B., and Milner, B. (1957). Loss of recent memory after bilateral hippocampal lesions. J. Neurol. Neurosurg. Psychiatry 20, 11-21. doi:10.1136/jnnp. 20.1.11

Shaw, S. M., Helmes, E., and Mitchell, D. (2006). Age-related change in visual, spatial and verbal memory. Australas. J. Ageing 25, 14-19. doi:10.1111/j.1741-6612. 2006.00134.x

Small, S. A., Tsai, W. Y., DeLaPaz, R., Mayeux, R., and Stern, Y. (2002). Imaging hippocampal function across the human life span: is memory decline normal or not? Ann. Neurol. 51, 290-295. doi:10.1002/ana.10105

St Jacques, P. L., Rubin, D. C., and Cabeza, R. (2012). Age-related effects on the neural correlates of autobiographical memory retrieval. Neurobiol. Aging 33, 1298-1310. doi:10.1016/j.neurobiolaging.2010.11.007

Staresina, B. P., and Davachi, L. (2006). Differential encoding mechanisms for subsequent associative recognition and free recall. J. Neurosci. 26, 9162-9172. doi:10.1523/JNEUROSCI.2877-06.2006

Thames, A. D., Foley, J. M., Wright, M. J., Panos, S. E., Ettenhofer, M., Ramezani, A., et al. (2012). Basal ganglia structures differentially contribute to verbal fluency: evidence from human immunodeficiency virus (HIV)-infected adults. Neuropsychologia 50, 390-395. doi:10.1016/j.neuropsychologia.2011.12.010

Troyer, A. K., D'Souza, N. A., Vandermorris, S., and Murphy, K. J. (2011). Agerelated differences in associative memory depend on the types of associations that are formed. Neuropsychol. Dev. Cogn. B Aging Neuropsychol. Cogn. 18, 340-352. doi:10.1080/13825585.2011.553273

Valenstein, E., Bowers, D., Verfaellie, M., Heilman, K. M., Day, A., and Watson, R. T. (1987). Retrosplenial amnesia. Brain 110(Pt 6), 1631-1646. doi:10.1093/brain/ 110.6.1631

West, M. J. (1993). Regionally specific loss of neurons in the aging human hippocampus. Neurobiol. Aging 14, 287-293. doi:10.1016/0197-4580(93)90113-P

Conflict of Interest Statement: The authors declare that the research was conducted in the absence of any commercial or financial relationships that could be construed as a potential conflict of interest.

Received: 28 May 2013; accepted: 20 December 2013; published online: 02 January 2014.

Citation: Dalton MA, Tu S, Hornberger M, Hodges JR and Piguet O (2014) Medial temporal lobe contributions to intra-item associative recognition memory in the aging brain. Front. Behav. Neurosci. 7:222. doi: 10.3389/fnbeh.2013.00222

This article was submitted to the journal Frontiers in Behavioral Neuroscience.

Copyright (c) 2014 Dalton, Tu, Hornberger, Hodges and Piguet. This is an open-access article distributed under the terms of the Creative Commons Attribution License (CC BY). The use, distribution or reproduction in other forums is permitted, provided the original author(s) or licensor are credited and that the original publication in this journal is cited, in accordance with accepted academic practice. No use, distribution or reproduction is permitted which does not comply with these terms. 


\section{APPENDIX}

Table A1 | Example list of words used in the intra-item associative memory task.

\begin{tabular}{|c|c|c|c|c|}
\hline \multicolumn{2}{|c|}{ Study } & \multicolumn{3}{|c|}{ Test } \\
\hline & & Identical & Recombined & Novel \\
\hline Bluebell & Hummingbird & Sandstorm & Bluebird & Underground \\
\hline Postcard & Trademark & Snowman & Postmark & Sunlight \\
\hline Playroom & Checkmate & Needlepoint & Playmate & Lapdog \\
\hline Fatherland & Neighborhood & Meatball & Fatherhood & Jumpsuit \\
\hline Downstairs & Waterfall & Brainpower & Downfall & Headdress \\
\hline Earthmover & Silkworm & Cupcake & Earthworm & Eyewitness \\
\hline Mainframe & Slipstream & Shuffleboard & Mainstream & Foresight \\
\hline Newspaper & Bandstand & Handbag & Newsstand & Keystone \\
\hline Beehive & Housekeeper & Flyscreen & Beekeeper & Armrest \\
\hline Hedgerow & Warthog & Boxcar & Hedgehog & Coalpit \\
\hline Backbone & Raindrop & Footstep & Backdrop & Hourglass \\
\hline Bottleneck & Paintbrush & Buttonhole & Bottlebrush & Spymaster \\
\hline Steamroller & Longboat & Motorcycle & Steamboat & Drumbeat \\
\hline Shortcut & Gingerbread & Courtship & Shortbread & Waistcoat \\
\hline Skydive & Mudlark & Vineyard & Skylark & Joystick \\
\hline \multicolumn{5}{|c|}{ WORDS USED AS IDENTICAL ITEMS AT TEST } \\
\hline Sandstorm & Buttonhole & & & \\
\hline Snowman & Boxcar & & & \\
\hline Needlepoint & Courtship & & & \\
\hline
\end{tabular}

